\title{
Changes of Sexual Behaviors in Rapamycin-injected Cichlid Fish Astatotilapia burtoni Males
}

\author{
Tae Ha Kim and ${ }^{\dagger}$ Young Chang Sohn \\ Dept. of Marine Molecular Bioscience, Gangneung-Wonju National University, Gangneung 25457, Korea
}

\begin{abstract}
Cichlid fish species exhibit characteristic sexual behaviors according to not only reproductive stages but also social status. In a reproductive season, Astatotilapia burtoni males compete for females and a small number of dominant winners finally obtain the chance of spermiation. In addition to the characteristic behaviors, the dominant males have relatively bigger gonadotropin-releasing hormone $1(\mathrm{GnRH} 1)$ neurons in the preoptic area (POA) of brain compared to those of subordinate males. Although the stimulatory effect of GnRH1 in vertebrate reproduction is well established, little is known about the triggering signal pathway to control GnRH1 neurons and GnRH1-mediated sexual behavior. In the present study, we evaluated the potential effect of TOR inhibitor rapamycin in relation to the cichlid male behaviors and GnRH1 neuron. After $14 \mathrm{~h}$ and $26 \mathrm{~h}$ of intraventricular injection of rapamycin, behavior patterns of chasing and courtship display did not show significant changes between rapamycin- and DMSO-injected males. Behaviors of spawning site entry increased in rapamycininjected fish at $26 \mathrm{~h}$ post-injection than at $14 \mathrm{~h}$ post-injection significantly $(P<0.05)$. Meanwhile, there was a tendency that GnRH1 neurons' soma size in the POA shrank by rapamycin injection, whereas the testes did not show notable changes. Taken together, these results suggest the possible role of TOR signal on GnRH1-mediated sexual behavior in cichlid dominant males, although further biological characterization of the TOR signaling pathway will be required to clarify this matter.
\end{abstract}

Key words : Cichlid, GnRH1, Rapamycin, Reproduction, Sexual behavior

\section{INTRODUCTION}

There are many examples of how environmental factors such as photoperiod and temperature, and social interactions such as aggression and meeting to the opposite sex, can alter reproductive status in sexually mature animals (Whittier et al., 1987; Wingfield et al., 1990; Hofmann \& Fernald, 2000; Taranger et al., 2003; Stevenson et al., 2008; Maruska $\&$ Fernald 2011a). These signals are mainly integrated in the brain and conveyed to the gonads through the highly conserved brain-pituitary-gonadal axis. This common signaling pathway begins at the gonadotropin-releasing hormone (GnRH1)-containing neurons located in the preoptic/hypothalamic area of the brain, whose projections to the pituitary gland control the release of gonadotropins responsible for gonad growth and steroid hormone production (Maruska \& Fernald 2011a; Jin et al., 2016).

The African cichlid fish, Astatotilapia burtoni, provides an interesting opportunity for analyzing neuronal changes because GnRH1 neurons in A. burtoni are remarkably plastic over short time scales in adult animals. GnRH1 neurons enlarge almost eight-fold and dramatically increase their

\footnotetext{
Manuscript received August 19, 2016, Received in revised form September 02, 2016, Accepted September 22, 2016

${ }^{\dagger}$ Corresponding Author : Young Chang Sohn, Dept. of Marine Molecular Bioscience, Gangneung-Wonju National University, Gangneung 25457, Korea. Tel/Fax : +82-33-640-2348, E-mail : ycsohn@gwnu.ac.kr

This is an Open Access article distributed under the terms of the Creative Commons Attribution Non-Commercial License (http:// creativecommons.org/licenses/by-nc/3.0) which permits unrestricted non-commercial use, distribution, and reproduction in any medium, provided the original work is properly cited.
} 
dendritic arbors in the fish that ascend in dominance status from non-territorial and non-reproductive subordinate males (S), to territorial and dominant reproductive states (D) (Francis et al., 1993). During the first 4 days of social ascent, for instance, GnRH1 neurons grow to their D size, increase GnRH1 production, and extend their dendritic arbors. Since male reproductive competence can be switched on or off depending on external influence, i.e., social environment, and is strongly regulated by the plasticity in the GnRH1 neuronal system, GnRH1 neurons in the cichlid could be used as a model to test hypotheses about which structural genes are involved in the dramatic and reversible changes in GnRH1 neuron size. However, the mechanisms and molecules involved in mediating these morphological changes in GnRH1 neurons remain unknown.

Adult cichlid males can rapidly and reversibly switch between $\mathrm{D}$ and $\mathrm{S}$ states depending on the composition of the social environment, and such transformations produce a distinct pattern of behavioral and physiological changes (Maruska \& Fernald 2013). When S males ascend in social status, they intensify their body coloration and increase dominance behaviors within minutes (Burmeister et al., 2005). Although they also described that even D males apparently began their typical aggressive behaviors anew each day, these data were sampled only during the 20 minutes following the first dominance behaviors because the newly ascended males were then sacrificed. As far as we know, little is known about how D behaviors change during days of social transition.

Target of rapamycin (TOR) was originally identified in yeast and later characterized in mammals (mTOR), structurally and functionally conserved mammalian counterpart (Schmelzle \& Hall, 2000). mTOR controls neuronal soma size, local protein synthesis in dendrites, axon growth and navigation, and synaptic plasticity (Kwon et al., 2003), as well as organization of the actin cytoskeleton, membrane traffic and protein degradation, protein kinase $\mathrm{C}$ signaling, ribosome biogenesis, and transcription (Schmelzle \& Hall, 2000). TOR is hypothesized to serve a conserved role in the control of cell growth (e.g., central controller of cell growth) and synaptic plasticity (Schmelzle \& Hall, 2000), but its function in non-mammalian vertebrates is relatively unclear. In addition, whether TOR controls neuron size in neuroendocrine cells like GnRH neurons remains unknown.

In the present study, we have microinjected rapamycin into the third ventricle of $\mathrm{D}$ male brains to understand how TOR inhibition affects the behavior of the animals. Furthermore, since rapamycin influences cytoskeletal rearrangement (Laplante \& Sabatini, 2012), we examined GnRH1 neuron size in the brain and germ cells in the testicular seminiferous tubules.

\section{MATERIALS AND METHODS}

\section{Fish maintenance and social manipulation}

Cichlid fish $A$. burtoni $(\mathrm{N}=50$; mixed sexes) purchased from AquaLab Co. (Daegu, Korea) were maintained in semirecirculating aquaria $(60 \times 30 \times 40 \mathrm{~cm})$ under environmental conditions $\left(25 \sim 27^{\circ} \mathrm{C}\right.$; constant aeration; $12 \mathrm{~h}$ light: $12 \mathrm{~h}$ dark cycle with full spectrum illumination by LED lighting (6,500 lux, Paisiz, Korea), and fed ornamental fish pellets (Tetrabits $+25 \%$, Fish Aquarium Home, India) twice a day. Aquaria contained corallite-covered bottoms with half terra cotta pots that served as spawning territories. All experimental fish were handled in accordance with the guideline, procedures and protocols of care and use of laboratory animals, Stanford Administrative Panel for Laboratory Animal Care.

\section{Behavioral analyses and intraventricular injection}

Adult D and S males (each one individual, body weight $5.2 \sim 5.7 \mathrm{~g}$, body length $5.0 \sim 6.0 \mathrm{~cm}$ ) were placed in the half side of fish tank separated by a black acrylic barrier with two adult females (body length $4.0 \sim 5.0 \mathrm{~cm}$ ) (Fig. 1). This compartment was isolated from each community group so that a group could not interact physically to the other one. 


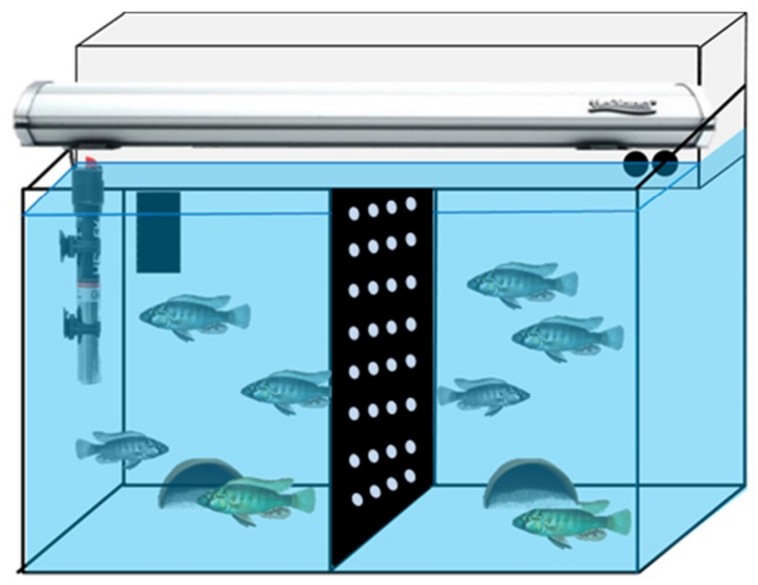

Fig. 1. Schematic representation of the experimental tank. Two Astatotilapia burtoni males expressing dominant behaviors were randomly selected and maintained in a half side of tank separated by a black acrylic barrier with two adult females and one subordinate male. The tank contains gravel-covered bottoms with half terra cotta pots as spawning territories.

At least two days ago, two community groups of fish were prepared for behavior monitoring. There are nine wellclassified behaviors in this cichlid fish species (Fernald, 1977). We have chosen three reproductive behaviors, i.e., chasing, courtship display and spawning site entry. Briefly, chasings define rapid swims of D male directed towards another female fish. Courtship displays were defined as rapid quivers by the male with presentation of the anal fin egg spots, and spawning site entries as the male entered half terra cotta pots as a spawning shelter (Maruska \& Fernald, 2013).

Experimental procedures for the intraventricular injection were generally performed according to a previous report for Nile tilapia Oreochromis niloticus (Ogawa et al., 2006). Briefly, fish were anesthetized in $0.01 \%$ solution of 3aminobenzoate methanesulfonate (MS-222; Omnilab, Bremen, Germany) and were then made a puncture on the scales and scull on top of the brain using a $20 \mathrm{G}$ needle. A $2 \mu \mathrm{L}$ Hamilton syringe (Hamilton, Nevada, USA) was stereotaxically injected into the third ventricle (stereotaxic $X, Y, Z$ coordinates for preoptic-GnRH1; 0.0/+2.5/+6.0 mm) for each individual. Rapamycin (LC Labs, Woburn, MA, USA) was diluted by dimethyl sulfoxide (DMSO) (Sigma-Aldrich, St. Louis, MO, USA) with methylene blue solution (Daejung Chem., Siheung, Korea). Final concentration of rapamycin solution was set to $20 \mu \mathrm{g} / \mu \mathrm{L}$ in $4 \%$ methylene blue solution and 1.0 $\mu \mathrm{L}$ was microinjected into third ventricle. In the control fish, the same volume of vehicle solution was injected into the brain. Rapamycin- and DMSO-injected groups $(n=3$ each group) were monitored for further two days in the fish tanks. Behaviors were recorded for 20 min for later quantification beginning at light onset (08:00 AM) using a digital video camera (FDR-AXP35, Sony, Japan) on the day before intraventricular injection and days 1-2 post-injection. Behavioral counts by video recordings were done without individual identification, i.e., rapamycin- and vehicle- injected fish.

\section{Histological observation of testes and $\mathrm{GnRH} 1$} neurons

Testes and brains were sampled from the rapamycinand DMSO-injected fish after final video recordings. Total body weight was obtained for each specimen examined followed by the removal and weighing of the testes to calculate GSI (gonad weight $\times 100 /$ body weight). Brains were removed following rapid cervical transection and testes were then fixed in $4 \%$ paraformaldehyde solution (Wako Pure Chem., Osaka, Japan) for histological examinations. Fragments of fixed brains and testes were embedded in paraffin and sectioned at a thickness of $7 \mu \mathrm{m}$. Serial sections for testes were affixed to glass slides, underwent clearing, hydration and finally stained with hematoxylin and eosin solution. Processed slides were examined using a light microscope (Nikon Eclipse E200, Nikon, Tokyo, Japan). For immunohistochemical observation, brain sections were rinsed in deionized water and phosphate buffered saline (PBS) three times. Nonspecific binding was blocked with a 
$2 \mathrm{~h}$ incubation in PBS supplemented with $0.2 \%$ bovine serum albumin (BSA), 10\% normal goat serum (Invitrogen, Grand Island, NY), and $0.3 \%$ Triton X-100. GnRH1 neurons were detected with a polyclonal guinea pig antibody for cichlid GnRH1 (Ma et al., 2015). Slides were incubated with the anti-GnRH1 antibody diluted 1:1,000 in PBS including $0.3 \%$ Triton $\mathrm{X}-100$ at $4{ }^{\circ} \mathrm{C}$. Following primary antibody incubation, brain slides were washed three times for $5 \mathrm{~min}$ in PBS and incubated for $2 \mathrm{~h}$ at RT with a goat anti-guinea pig IgG HRP (Santa Cruz Biotech., Dallas, TX, USA) diluted 1:500 in PBS. After rinsing in PBS including $0.3 \%$ Triton $\mathrm{X}-100$ three times, slides were treated with DAB-peroxidase substrate solution (1\% DAB, $0.015 \%$ $\mathrm{H}_{2} \mathrm{O}_{2}, 0.01 \mathrm{M}$ PBS, $\mathrm{pH}$ 7.2) for $10 \mathrm{~min}$. Slides were then washed three times for $5 \mathrm{~min}$ in PBS and cover-slipped with a mounting medium.

\section{Statistical analysis}

The data represent means \pm SEM. The data were analyzed using one-way analysis of variance (ANOVA) and Student's $t$ test using SPSS software (v. 23). In all cases, $P$ values $<$ 0.05 were set to indicate statistical significance.

\section{RESULTS}

\section{Reproductive behaviors of $D$ males}

When the lights come on in the examination tank, subjects show a rapid and steady increase in rates of dominance behaviors, such as chasing, courtship displays, and spawning site entries, that typically reached $5 \sim 10$ behaviors $/ \mathrm{min}$ after light onset (Maruska \& Fernald, 2010). In the present study, we also observed the typical behaviors, suggesting that the D males displayed a normal range of intrinsic performance. Chasing behaviors in rapamycin-injected fish did not show significant changes compared to that of DMSOinjected fish by $26 \mathrm{~h}$ post-injection (Fig. 2). Courtship display in rapamycin-injected fish slightly decreased at 14 $\mathrm{h}$ but increased at $26 \mathrm{~h}$ post-injection when it was compared

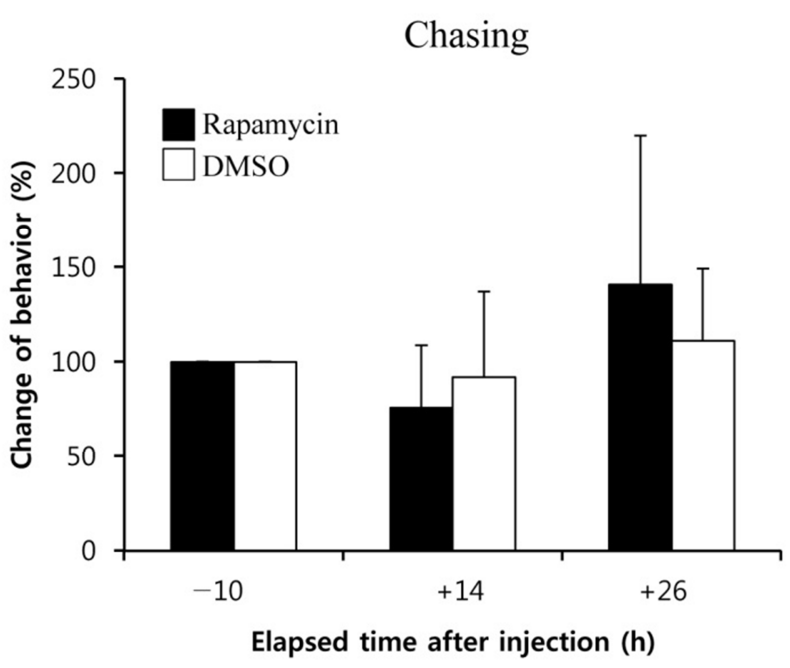

Fig. 2. Changes in chasing behavior of cichlid dominant males. Behavior was recorded by a digital video camera at each morning (08:00 AM) with light onset, counted for $20 \mathrm{~min}$ later, and expressed as relative percentages compared to those of non-injected animals $(-10 \mathrm{~h})$. After $10 \mathrm{~h}$ of the first day recording, intraventricular injection was done with rapamycin $(20 \mu \mathrm{g} / \mu \mathrm{L})$ or same volume of DMSO $(0 \mathrm{~h})$ and fish was further maintained up to $+26 \mathrm{~h}$. Data are plotted as mean $\pm \operatorname{SEM}(\mathrm{n}=3)$.

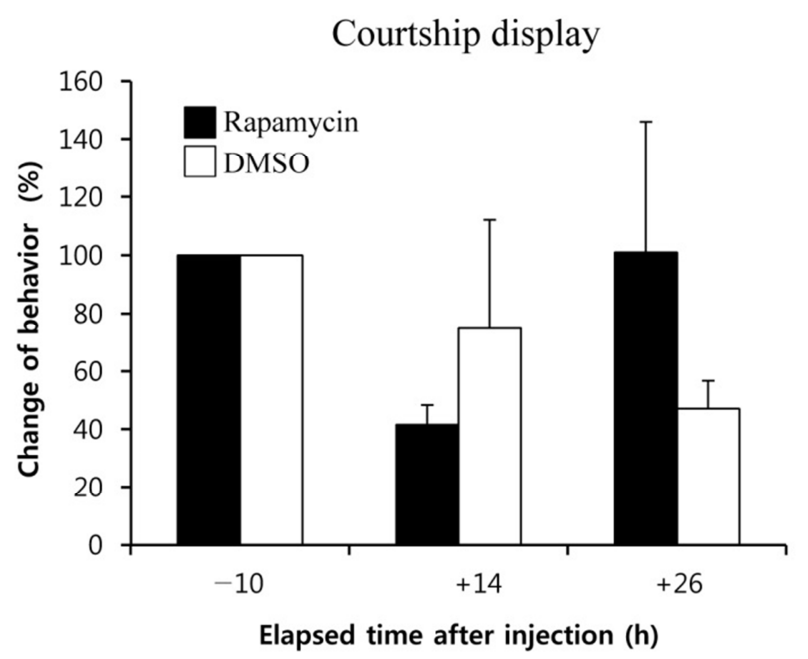

Fig. 3. Changes in courtship display of cichlid dominant males. Behavior was counted for three consecutive morning (08:00 AM) for 20 min with light onset and expressed as relative percentages compared to those of non-injected animals ( $-10 \mathrm{~h})$. Experimental procedures and behavioral analysis are noted in Fig. 2. 


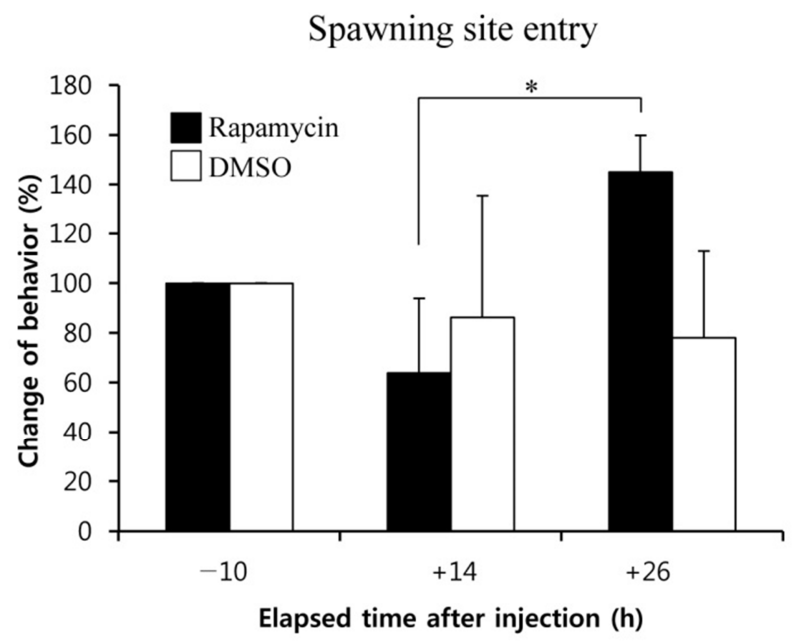

Fig. 4. Changes in spawning site entry behavior of cichlid dominant males. Behavior was counted for three consecutive morning (08:00 AM) for 20 min with light onset and expressed as relative percentages compared to those of non-injected animals $(-10 \mathrm{~h})$. Experimental procedures and behavioral analysis are noted in Fig. 2. Asterisk indicates significant difference $(P<0.05)$

to that of control fish, although the differences were not significant (Fig. 3). Behavior rates of spawning site entry increased in rapamycin-injected fish at $26 \mathrm{~h}$ post-injection than at $14 \mathrm{~h}$ post-injection significantly $(P<0.05)$, whereas the DMSO-injected control fish did not show any change during experimental examination (Fig. 4).

\section{Immunohistochemical observation of hypothalamic}

\section{GnRH1 neurons}

We examined the GnRH1 neurons in the POA to judge whether the behaviors of the rapamycin-injected fish are dependent on the neuronal activities. The soma sizes of GnRH1 neurons in fish injected with rapamycin showed a tendency of decreasing compared to those of DMSOinjected fish (Fig. 5).

\section{Examination of testes}

There was no significant difference of GSI between the rapamycin- and DMSO-injected fish (0.69 0.95). Histological

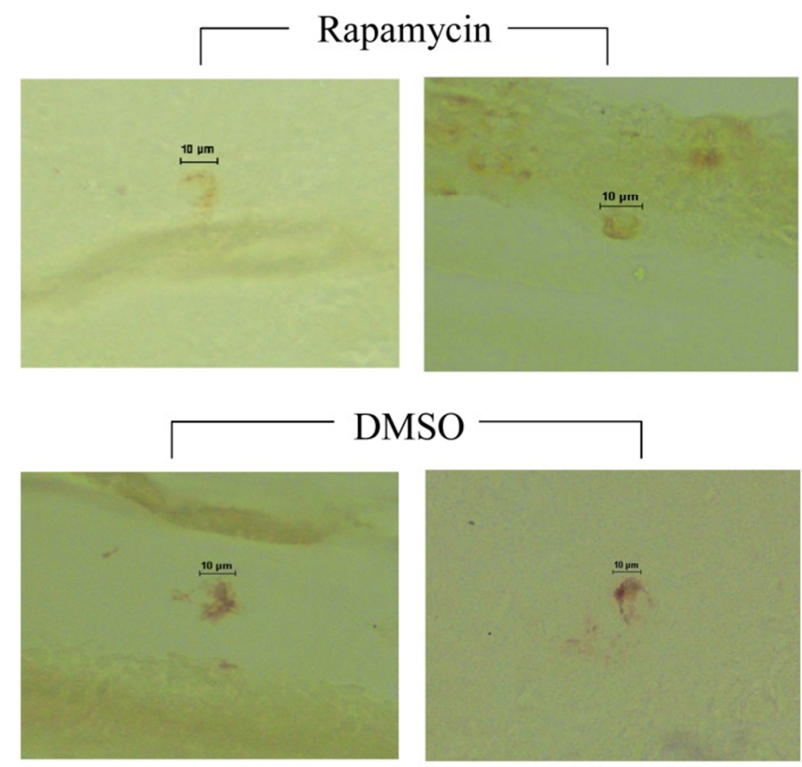

Fig. 5. Representative GnRH1-immunoreactive neurons of cichlid dominant male brains. Rapamycin- or DMSO-injected fish were sacrificed and the brains were applied to an immunohistochemical examination. GnRH1 neurons in the preoptic areas of brains with were detected with a polyclonal guinea pig antibody for cichlid GnRH1 and detailed procedures are described in Materials and Methods. Scale bars indicate $10 \mu \mathrm{m}$.

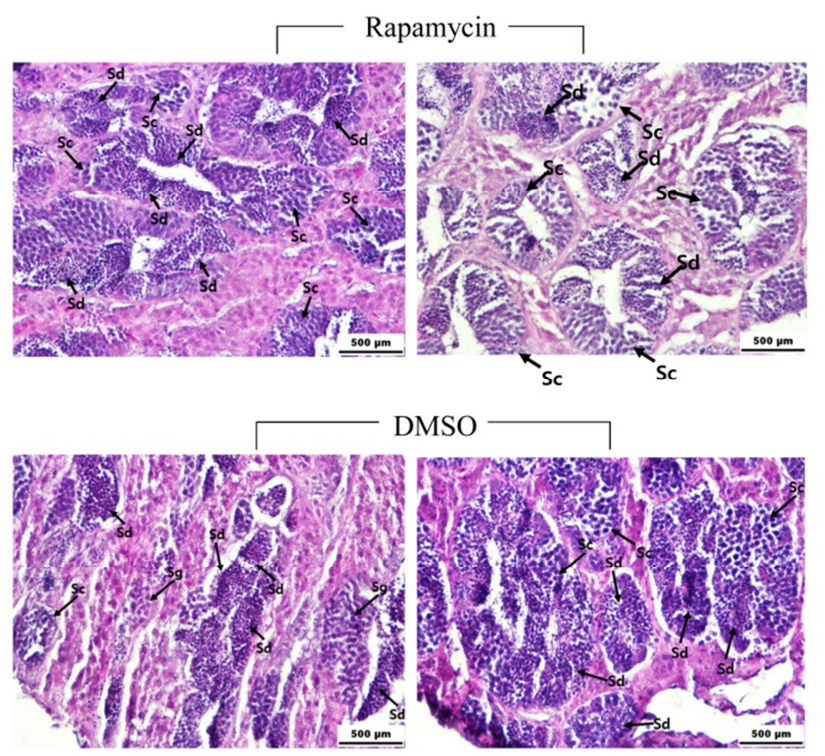

Fig. 6. Representative testes of cichlid dominant males. The testes of rapamycin- or DMSO-injected fish were fixed and examined by routine hematoxylin and eosin staining method. Scale bars indicate $500 \mu \mathrm{m}$. Sg, spermatogonia; Sc, spermatocyte; sd, spermatid. 
observations showed that the majority of the germ cells in the testes of rapamycin- and DMSO-injected males are spermatocytes and spermatids (Fig. 6). However, the most advanced spermatogenetic cells, e.g., mature spermatozoa, were not detected in either rapamycin- or DMSO-injected fish.

\section{DISCUSSION}

A. burtoni has become a valuable vertebrate model species for studying social environment influences on behavior, the brain, and reproductive plasticity (Fernald, 1977; Maruska $\&$ Fernald, 2010, 2013). The males of this species exist in two distinct phenotypes, non-reproductive subordinate $\mathrm{S}$ and dominant reproductive $\mathrm{D}$ types. The $\mathrm{D}$ males of yellow and blue coloration have territories that they defend strongly and spend considerable time attempting to court with matured females, whereas the $\mathrm{S}$ males without territories school with females and other $\mathrm{S}$ males and flee from aggressive D males (Fernald, 1977; Maruska \& Fernald, 2010). Although these social behaviors are evidently linked to hypothalamic neurons expressing GnRH1, pituitary gonadotrophic and testicular activities, (Maruska \& Fernald, 2011b), the detailed mechanisms and upstream molecules involved in mediating these social behaviors are largely unknown. In the present study, TOR inhibitor rapamycin was monitored as a candidate responsible for sexual behavior and GnRH1 neuron enlargement in male $A$. burtoni.

Our data show that intraventricular injection of rapamycin has only a marginal impact on the social and reproductive behaviors of the D males. As mentioned by Maruska and Fernald (2010), three distinctive male behaviors, chasing (51 165 times/20 min), courtship display (15 86), and spawning site entry (11 33), were continuously shown in the aggressive D males during this experiment. Although spawning site entry slightly increased in rapamycin-injected fish at $26 \mathrm{~h}$ post-injection, other behaviors did not show significant changes between experimental and control groups. In male mice, inhibition of hypothalamic mTOR signaling pathway by rapamycin regulates sympathetic nerve traffic and behavioral rhythms (Muta et al., 2015), even though mTOR generally operates as sensor of cellular energy status and effector for its coupling to cell growth and proliferation (Schmelzle \& Hall, 2000). Since the final concentration of rapamycin solution $(20 \mu \mathrm{g} / \mu \mathrm{L})$ injected into brains is not lower compared to other experiments in rats $(50 \mu \mathrm{g} / \mu \mathrm{L})$ when the body mass is considered (Hebert et al., 2014), it is likely that $\mathrm{D}$ males' dominant behaviors would not be affected by TOR inhibitor within a short-term period.

Increased soma size of GnRH1 neurons in D males than that of $\mathrm{S}$ males provides an important clue to understand the social and reproductive behaviors in this species (Francis et al., 1993). Although the activity of GnRH1 neurons is crucial for the pituitary and gonadal regulatory mechanisms, the regulators of GnRH1 neuron activities are still poorly understood in non-mammalian species (Karigo \& Oka, 2013). Some of earlier experiments using neuropeptides and neurotransmitters such as neuropeptide Y, noradrenaline, serotonin, and dopamine demonstrated their activities on GnRH1 neurons by measuring the amount of GnRH1 peptide released in fish species both in vitro and in vivo examination (Zohar et al., 2010). Further, in primate pituitary rapamycin treatment abolished a stimulatory effect of kisspeptin on gonadotropin secretion (Luque et al., 2011), suggesting that the stimulation of pituitary gonadotropin elicited by kisspeptin is mediated by mTOR. In the present study, we suggested that the mTOR signaling cascade may mediate GnRH1 neuronal activity in cichlid D males because a tendency of decreasing soma size of GnRH1 neurons was shown in D males received an intraventricular injection of rapamycin. To determine whether the mTOR signal pathway is closely linked to GnRH1 neuron activity, a series of fine-tuned in vivo and in vitro experiments should be performed. The developmental stages of testes did not show marked 
changes between rapamycin- and vehicle-injected fish. The majority of the seminiferous tubules in both groups' testes were filled with spermatocytes and spermatids, suggesting that the behaviors of $\mathrm{D}$ males, i.e., chasing, courtship display, and spawning site entry, are not likely associated with actual spermiation. With regard to aggressive behaviors of reproductively active cichlid and zebrafish males, testosterone secretion was considered as a promising trigger of the characteristics (Maruska \& Fernald, 2010; Teles \& Oliveira, 2016). Thus, testosterone level could be a good indicator for mTOR-mediated reproductive activities and behaviors in $\mathrm{D}$ cichlid males.

\section{ACKNOWLEDGEMENTS}

We thank Russell D. Fernald for his kind supplying the GnRH1 antibody. This research was supported by Basic Science Research Program through the National Research Foundation of Korea (NRF) funded by the Ministry of Education (2012R1A1A2044506) and by Gangneung-Wonju National University.

\section{REFERENCES}

Burmeister SS, Jarvis ED, Fernald RD (2005) Rapid behavioral and genomic responses to social opportunity. PLoS Biol 3:e363.

Fernald RD (1977) Quantitative behavioral observations of Haplochromis burtoni under semi-natural conditions. Anim Behav 25:643-653.

Francis RC, Soma K, Fernald RD (1993) Social regulation of the brain-pituitary-gonadal axis. Proc Natl Acad Sci USA 90:7794-7798.

Hebert M, Licursi M, Jensen B, Baker A, Milway S, Malsbury C, Grant VL, Adamec R, Hirasawa M, Blundell J (2014) Single rapamycin administration induces prolonged downward shift in defended body weight in rats.
PLoS One 9:e93691.

Hofmann HA, Fernald RD (2000) Social status controls somatostatin neuron size and growth. Neuroscience 20:4740-4744.

Jin YH, Park JW, Kim JH, Kwon JY (2016) Neurokinin Brelated peptide suppresses the expression of GnRH I, Kiss 2 and tac 3 in the brain of mature female Nile tilapia Oreochromis niloticus. Dev Reprod 20:51-61.

Karigo T, Oka Y (2013) Neurobiological study of fish brains gives insights into the nature of gonadotropin-releasing hormone 1-3 neurons. Front Endocrinol (Lausanne) 4:177.

Kwon CH, Zhu X, Zhang J, Baker SJ (2003) mTOR is required for hypertrophy of Pten-deficient neuronal soma in vivo. Proc Natl Acad Sci USA 100:1292312928.

Laplante M, Sabatini DM (2012) mTOR signaling in growth control and disease. Cell 149:274-293.

Luque RM, Córdoba-Chacón J, Gahete MD, Navarro VM, Tena-Sempere M, Kineman RD, Castaño JP (2011) Kisspeptin regulates gonadotroph and somatotroph function in nonhuman primate pituitary via common and distinct signaling mechanisms. Endocrinology 152:957-966.

Ma Y, Juntti SA, Hu CK, Huguenard JR, Fernald RD (2015) Electrical synapses connect a network of gonadotropin releasing hormone neurons in a cichlid fish. Proc Natl Acad Sci USA 112:3805-3810.

Maruska KP, Fernald RD (2010) Behavioral and physiological plasticity: rapid changes during social ascent in an African cichlid fish. Horm Behav 58:230-240.

Maruska KP, Fernald RD (2011a) Social regulation of gene expression in the hypothalamic-pituitary-gonadal axis. Physiology 26:412-423.

Maruska KP, Fernald RD (2011b) Plasticity of the reproductive axis caused by social status change in an African cichlid fish: II. testicular gene expression and spermatogenesis. Endocrinology 152:291-302.

Maruska KP, Fernald RD (2013) Social regulation of male 
reproductive plasticity in an African cichlid fish. Integr Comp Biol 53:938-950.

Muta K, Morgan DA, Rahmouni K (2015) The role of hypothalamic mTORC1 signaling in insulin regulation of food intake, body weight, and sympathetic nerve activity in male mice. Endocrinology 156:1398-1407.

Ogawa S, Akiyama G, Kato S, Soga T, Sakuma Y, Ishwar SP (2006) Immunoneutralization of gonadotropin-releasing hormone type-III suppresses male reproductive behavior of cichlids. Neurosci Lett 403:201-205.

Schmelzle T, Hall MN (2000) TOR, a central controller of cell growth. Cell 103:253-262.

Stevenson TJ, Bentley GE, Ubuka T, Arckens L, Hampson E, MacDougall-Shackleton SA (2008) Effects of social cues on GnRH-I, GnRH-II, and reproductive physiology in female house sparrows (Passer domesticus). Gen Comp Endocrinol 156:385-394.

Taranger GL, Vikingstad E, Klenke U, Mayer I, Stefansson
SO, Norberg B, Hansen T, Zohar Y, Andersson E (2003) Effects photoperiod, temperature and GnRHa treatment on the reproductive physiology of Atlantic salmon (Salmo salar L.) broodstock. Fish Physiol Biochem 28:403-406.

Teles MC, Oliveira RF (2016) Androgen response to social competition in a shoaling fish. Horm Behav 78:8-12.

Whittier JM, Mason RT, Crews D, Licht P (1987) Role of light and temperature in the regulation of reproduction in the red-sided garter snake, Thamnophis sirtalis parietalis. Can J Zool 65:2090-2096.

Wingfield JC, Hegner R, Dufty A Jr, Ball GF (1990) The "challenge hypothesis": theoretical implications for patterns of testosterone secretion, mating system, and breeding strategies. Am Nat 136:829-846.

Zohar Y, Muñoz-Cueto JA, Elizur A, Kah O (2010) Neuroendocrinology of reproduction in teleost fish. Gen Comp Endocrinol 165:438-455. 